\title{
THE EFFECT OF ADDITION SILICA FUME, SUPERPLASTICIZER, AND BONDING TO REACHING HIGH QUALITY LIGHTWEIGHT CONCRETE
}

\author{
Ayuk Putri Yuliansari \\ Faculty Engineering \\ Narotama University Surabaya \\ ayukputriy@gmail.com \\ Fredy Kurniawan \\ Faculty Engineering \\ Narotama University Surabaya \\ fredy@narotama.ac.id
}

\begin{abstract}
In this research, a lightweight concrete mixture was made with foam and made use of the remaining broken rock from the Crusher Machine process, which was washed ashes. Other added ingredients used in the mixture are Silica Fume, Superplasticizer (Admixture Type F) and Bonding. This reseach uses a mix design from Circular Letter Number: 44 / SE / M / 2015 Concerning Design Guidelines for Mixing Light Material with Foam Mortar for Road Construction, Ministry of PUPR. Trial mix variations carried out are in proportion (SF 5\%; BO $0.5 \%$ ), (SF 10\%; BO 0.5\%), (SF 5\%; BO 1\%), (SF 10\%; BO 1\%), as well as foam agents with variations 1:20 and 1:40. The treatment process (curing) of the test object is immersed in a water bath, and the testing of the compressive strength of the test object is carried out at the age of 7 days, 14 days and 28 days. By using stone ash, lightweight concrete was obtained with an average specific gravity of $1722 \mathrm{~kg} / \mathrm{m}^{3}$ to $1884 \mathrm{~kg} / \mathrm{m}^{3}$. From the research results obtained the highest average compressive strength with the percentage of material that is in the variation of the SF $10 \%$; BO $0.5 \%$ and ratio foam (1) : water (40) only for $\mathrm{K}$ $190.6 \mathrm{~kg} / \mathrm{cm}^{2}$ or FC $15.82 \mathrm{MPa}$. In each specimen the value of quality efficiency with the most optimum weight variation at SF $10 \%$; BO $0.5 \%$ with a coefficient value of $10.03 \%$.
\end{abstract}

Keywords: lightweight concrete, foam, stone ash, silica fume, superplasticizer, and bonding.

\section{INTRODUCTION}

Concrete is one of the most important elements in building structures. Indonesia is an archipelago that is developing infrastructure development and almost all construction uses concrete. According to SNI 2847: 2013, concrete is a mixture of portland cement or other hydraulic cement, fine aggregate, coarse aggregate, and water with or without additives. The development of science and technology has led to the emergence of various kinds of innovations in the field of construction. The types of concrete in construction are very varied, can be reinforced concrete, prestressed concrete, mortar concrete, pre-cast concrete, and lightweight concrete.

Lightweight concrete based on unit weight (SNI 03-2847-2002) is concrete which has a density or unit weight $<1,900 \mathrm{~kg} / \mathrm{m}^{3}$ consisting of cement, sand, water, foam agent, or other added materials. Lightweight concrete consists of two types, namely Autoclaved Aerated Concrete (AAC) and Cellular Lighweight Concrete (CLC), lightweight concrete with AAC type in its mixing using aluminum paste while for CLC lightweight concrete in mixing using foam.

Foam concrete material in cellular lightweight concrete is one of the construction materials that has been developed lately. The thing that should be considered in the 
manufacture of cellular lightweight concrete is the use of foam agents that function to trap voids in mortars, so that dry densities between $500 \mathrm{~kg} / \mathrm{m}^{3}-1600 \mathrm{~kg} / \mathrm{m}^{3}$ are obtained (Jitchaiyaphum, K, et. Al, 2011 : 1157). The mixture in cellular lightweight concrete is cement, fine aggregate, water, foam agent and other added materials needed in its manufacture. Fine aggregate used is Abu Batu, which utilizes the results of the rest of the process of breaking the stone with a Crusher Machine.

Additives Type F, Silica Fume and Bonding are other added ingredients to achieve highquality cellular lightweight concrete. Type $\mathrm{F}$ additives are used to obtain the desired level of consistency or specifications by reducing water weight by $>12 \%$. Bonding is a mixture of concrete adhesives that serves to increase the adhesion of concrete so that the concrete becomes solid and air cavities are reduced. Silica fume is a very fine pozolan material and partly consists of silica element, which is produced from a furnace as a by-product of the silicon metal industry (ASTM C 1240-93). Silica fume is physically finer than cement and chemically contains a high $\mathrm{SiO} 2$ element, which increases the strength of concrete when used as an additive to concrete.

In accordance with the description in the research background above, the writer can formulate the problem to be discussed :

1. Is using stone ash and foam agent able to produce lightweight concrete?

2. Is the addition of Silica Fume, Type F additives and bonding able to affect the quality and achieve high quality lightweight concrete?

3. What is the compressive strength produced in concrete with the addition of Silica Fume, Type $F$ additives and bonding?

\section{LITERATURE REVIEW}

High quality lightweight concrete is concrete which has a specific gravity ranging from $350 \mathrm{~kg} / \mathrm{m}^{3}-1900 \mathrm{~kg} / \mathrm{m}^{3}$ with compressive strength exceeding $6000 \mathrm{psi}$ (40 Mpa). Lightweight Concrete Mixture Material According to the Ministry of Public Works and Public Housing Design Guidelines for Mixing Lightweight Mortar-Foam Materials for Road Construction, lightweight concrete using a mixture of Portland cement or other hydraulic cement, fine aggregate, foam, and water, with or without additives (admixture).

Fine Aggregate is an aggregate of not more than $5 \mathrm{~mm}$ in size, so it can be natural sand or sand from rock breaking resulting from rock breaking (stone ash). According to (SNI 036819-2002) the grains produced by fine aggregates must be hard and durable, must not contain mud, clay and loose material / more easily destroyed by more than $5 \%$. Fine aggregate must be free from charcoal, wood objects and other unwanted impurities.

According to the Indonesian Industrial Standards (SII. 0013 - 81) Cement is a hydraulic cement produced by smoothing clinkers which mainly consists of calcium silicates which are hydrolyzed together with additives which are usually used in casts. The composition of materials in cement consists of Silicondoxide (SiO), Aluminumoxide (AIO), Calciumoxide $(\mathrm{CaO})$, Magnesiumoxide $(\mathrm{MgO})$, Sulfurtrioxide $(\mathrm{SO})$, Sodium Oxide $(\mathrm{NaO})$.

Foam is a foam liquid containing vegetable protein or the like that can produce stable separate bubbles so as to produce a mixture of lightweight materials that meet technical specifications. Foam making material is foam agent and water, to make foam, mixing foam fluid and water using a foam generator and compressor.

According to the standard "Specification for Silica Fume fauna Use in Hydraulic Cement Concrete and Mortal" (ASTM.C.1240.1995: 637-642), silica fume is a smooth pozzolan material, where more silica composition is produced from higher kilns or residual production silicon or silicon iron alloy (known as a combination of micro silica with silica fume). The use of silica fume is always the same as High Range Water Reducer (Superplasticizer). Due to 
the use of water in concrete and the presence of silica fume which fills the pores and is pozzolanic, it causes the concrete to become impermeable, durable and of high strength.

According to ASTM C 494 and British Standard 5075, Superplasticizer is a highly effective water-reducing chemical additive. With the use of these additives obtained a mixture with a lower cement water factor at the same mortar viscosity value or obtained with a thinner viscosity with the same cement water factor, so that the compressive strength of concrete is higher. Superplasticizer also has a great influence in increasing workability.

\section{Material Test Analysis}

Analysis of cement specific gravity (ASTM C 188 - 89)

Objective: Determine the specific gravity of cement.

How to calculate :

Cement Weight $=(\mathrm{w} 1) \ldots$. gram

Cement weight + oil + measuring flask $=(w 2) \ldots .$. grams

Measuring pumpkin weight + oil $=($ w3 $) \ldots .$. grams

Then $\mathrm{Bj}==\frac{0,8 \mathrm{w} 1}{(\mathrm{w} 1+\mathrm{w} 3-\mathrm{w} 2)}$

Cement Volume Weight Analysis (ASTM C 188 - 89)

Objective: Determine the volume weight of cement in a loose or bound state.

How to calculate :

Cylinder weight $=(\mathrm{w} 1) \ldots$. gram

Cylinder weight + cement $=($ w2 $) \ldots .$. gram

Cement weight $=(\mathrm{w} 2-\mathrm{w} 1) \ldots . . \mathrm{gram}$

Cylinder volume $=(\mathrm{v}) \ldots . . \mathrm{dm} 3$ (liter)

Then the volume weight $=$

\section{Sieve analysis (ASTM C 136 - 95A)}

Objective: To determine the grain size distribution / gradation of stone ash.

Calculation Method:

Calculative cumulative weight retained $=$ cumulative retained weight of the filter above + the amount of retained weight of the filter underneath.

Percentage on hold $=\frac{\text { Komulatif berat tertahan }}{\text { Berat contoh }} \times 100 \%$

\section{Analysis of specific gravity (ASTM C 128 - 93)}

Objective: Determine the specific gravity of stone ash.

Calculation Method:

Weight of pumpkin + stone ash + water $=(w 1)$

Stone ash weight $=500$

Pumpkin weight + water $=($ w2)

Then the specific gravity of stone ash $=\frac{500}{(500+w 2)-w 1}$

\section{Analysis of rock ash absorption (ASTM C 128 - 93)}

Objective: To determine the water content of rock ash infiltration.

Calculation Method:

SSD sand weight $=500$

Sand weight (oven) $=($ w1 $)$

Then the rate of absorption of sand $=\frac{500-\mathrm{w} 1}{\mathrm{w} 1} \times 100 \%$ 
Analysis of Cleanliness of Stone Ash on Organic Materials (ASTM C 40 - 92)

Objective: Determine the levels of organic matter in the aggregates used in concrete mix.

How to experiment levels of organic substances:

The volume of stone ash +200 grams was given a $\mathrm{NOH}$ solution of $3 \%$, shaken for 10 minutes and allowed to stand for 24 hours.

\section{Analysis of Ash Stone Cleanliness for Mud (Washing) [ASTM C 117 - 95]}

Objective: To determine the amount of mud content in rock ash.

How to count:

Dry sand weight $=(w 1) \ldots .$. gram

Weight of dry clean sand $=(w 2) \ldots .$. gram

Mud Content $==\frac{(\mathrm{w} 1-\mathrm{w} 2)}{\mathrm{w} 1} \times 100 \%$

Rock Ash Volume Weight Analysis (ASTM C 29 / C 29 M - 91)

Objective: Determine the volume weight of stone ash in both loose and solidconditions.

How to calculate :

Cylinder weight $=(\mathrm{w} 1) \ldots \mathrm{kg}$

The weight of the cylinder + sand $=(w 2) \ldots \mathrm{kg}$

Sand weight $=(\mathrm{w} 2-\mathrm{w} 1) \ldots \mathrm{kg}$

Cylinder volume $=(\mathrm{v}) \ldots$ liters $(\mathrm{dm} 3)$

Volume weight $=$

Treatment / curing in lightweight concrete that is immersed in a tub / curing tub and testing is carried out for 7 days, 14 days and 28 days. Concrete compressive strength value is determined when the concrete reaches the age of 28 days. The strength of concrete will increase rapidly until the age of 28 days, but after that the increase is not too significant. Generally at the age of 7 days the compressive strength reaches $65 \%$ and at the age of 14 days reaches $88 \%$ - $90 \%$ of the compressive strength of the concrete age of 28 days.

For the calculation of compressive strength of test specimens according to SNI 031974-2011 article 7, using systematic calculations can be written as follows:

- Concrete Compressive Strength: $f c^{\prime}=\frac{\mathrm{P}}{\mathrm{A}}$

Where :

$\mathrm{P}=$ Axial Compressive Force $(\mathrm{N})$

$A=$ Test area $(\mathrm{mm} 2)$ cross-sectional area

$\mathrm{fc}{ }^{\prime}=$ Concrete compressive strength $(\mathrm{N} / \mathrm{mm} 2)$

- Average Compressive Strength: $\mathrm{fcr}=\quad$ fcr $=\sum_{t=1}^{n} f c i: n$

Where :

$\mathrm{P}=$ Axial Compressive Force $(\mathrm{N})$

$\mathrm{A}=$ Test material cross-sectional area $(\mathrm{mm} 2)$

fc ' $=$ Concrete compressive strength $(\mathrm{N} / \mathrm{mm} 2)$

$\mathrm{fcr}=$ Average compressive strength of concrete $(\mathrm{N} / \mathrm{mm} 2)$

$\mathrm{fci}=$ concrete compressive strength obtained from the test results $(\mathrm{N} / \mathrm{mm} 2)$

$\mathrm{n}=$ Number of test specimens 


\section{RESEARCH METHODOLOGY}

\section{Time and Place of Research}

Research time is planned within a period of 6 months from August 2019 - January 2020. The research was conducted at the Concrete Laboratory of PT. Varia Usaha Beton Gresik, East Java.

\section{Preparation of Materials and Tools}

The materials used in this research are:

a) Type 1 OPC cement produced by PT. Semen Indonesia;

b) Fine aggregate in the form of Abu Batu imported from Pandaan District, Pasuruan Regency, East Java;

c) Silica Fume comes from PT Sika Indonesia;

d) Foam comes from PT Erta Tekno Indonesia;

e) The added ingredients used are: -Superplasticizer or often called Type F production of PT. Concrete Varia Enterprises -Bonding came from PT Erta Tekno Indonesia

f) Water used for making concrete water that is clean and contains no chemicals.

\section{Material Testing}

Fine Aggregate Testing (Stone Ash) in the form of:

1. Fine aggregate filter analysis, based on (ASTM C $136-95 A)$

2. Fine aggregate specific gravity analysis, based on (ASTM C 128 - 93)

3. Analysis of fine aggregate infiltration, based on (ASTM C 128 - 93)

4. Fine aggregate hygiene analysis of organic materials, based on (ASTM C 40 - 92)

5. Sand Cleanliness Analysis for Mud by (Washing) method, based on (ASTM C 117 - 95)

6. Weight Analysis of fine aggregate sand volume, based on (ASTM C 29 / C 29 M - 91)

\section{Mix Design Planning}

In this research the mix design planning uses Circular Letter Number: 44 / SE / M / 2015 concerning Guidelines for Designing Mixed Mortar-Foam Light Material for Road Construction, Ministry of Public Works and Public Housing.

\section{Target Concrete Specific Gravity, Foam \& Slump Flow Test Specific Gravity}

The target specific gravity is $<1900 \mathrm{~kg} / \mathrm{m}^{3}$, the target specific gravity foam $(0.055-0.085 \mathrm{t} /$ $\mathrm{m}^{3}$ ), and the target flow mortar foam $180 \pm 200 \mathrm{~mm}$.

\section{Trial Mix Process and Manufacture of Test Objects}

In this study the making of test specimens using cylinders $15 \mathrm{~cm} \times 30 \mathrm{~cm}$, with a total of 4 trial variations and each variation amounted to 9 test specimens.

\section{Table 1.}

Trial Variation and Number of Test Objects

\begin{tabular}{|c|c|c|c|c|c|}
\hline \multirow{2}{*}{ Kode } & \multirow{2}{*}{ No } & \multicolumn{3}{|c|}{$\begin{array}{c}\text { Uji Kuat Tekan } \\
\text { (KN) }\end{array}$} & \multirow{2}{*}{ KET } \\
\hline & & $\begin{array}{c}7 \\
\text { Hari }\end{array}$ & $\begin{array}{c}14 \\
\text { Hari }\end{array}$ & $\begin{array}{c}28 \\
\text { Hari }\end{array}$ & \\
\hline \multirow{3}{*}{ Normal } & 1 & & & & \multirow{3}{*}{ Normal } \\
\hline & 2 & & & & \\
\hline & 3 & & & & \\
\hline \multirow{3}{*}{$\begin{array}{c}\text { BRMT } \\
1\end{array}$} & 1 & & & & \multirow{3}{*}{$\begin{array}{c}\text { SF } \\
5 \%+B O \\
0,5 \% \\
\end{array}$} \\
\hline & 2 & & & & \\
\hline & 3 & & & & \\
\hline
\end{tabular}




\begin{tabular}{|c|c|c|}
\hline \multirow{3}{*}{$\begin{array}{c}\text { BRMT } \\
2\end{array}$} & 1 & \multirow{3}{*}{$\begin{array}{c}\text { SF } \\
10 \%+B O \\
0,5 \%\end{array}$} \\
\hline & 2 & \\
\hline & 3 & \\
\hline \multirow{3}{*}{$\begin{array}{c}\text { BRMT } \\
3\end{array}$} & 1 & \multirow{3}{*}{$\begin{array}{c}\text { SF } \\
5 \%+B O \\
1 \%\end{array}$} \\
\hline & 2 & \\
\hline & 3 & \\
\hline \multirow{3}{*}{$\begin{array}{c}\text { BRMT } \\
4\end{array}$} & 1 & \multirow{3}{*}{$\begin{array}{c}\text { SF } \\
10 \%+B O \\
1 \%\end{array}$} \\
\hline & 2 & \\
\hline & 3 & \\
\hline
\end{tabular}

\section{Concrete Treatment of Test Objects (Curing)}

Concrete curing / maintenance aims to maintain and guarantee the quality of the concreting. In this study the steps taken are as follows:

Dismantling the test specimen is done \pm 24 hours after making it;

The specimen is immersed until the age of 7 days, 14 days and 28 days;

The test object is taken / dried the day before the test day.

\section{Concrete Compressive Strength Testing}

Press test procedure:

The test object is placed on a press machine sentries;

The machine is running, press with a constant increase in load ranging from 2 to $4 \mathrm{~kg} / \mathrm{cm} 3$ per second.

\section{RESULTS AND DISCUSSION \\ Table 2}

Volume Weight Analysis (ASTM C 29 / C 29 M-91)

\begin{tabular}{|l|c|c|c|c|}
\hline \multicolumn{1}{|c|}{ PERCOBAAN } & SATUAN & LEPAS & GOYANG & ROJOKAN \\
\hline Berat Moulding (W1) & $\mathrm{gr}$ & 3800 & 3800 & 3800 \\
\hline $\begin{array}{l}\text { Berat Moulding + Abu } \\
\text { Batu (W2) }\end{array}$ & $\mathrm{gr}$ & 8100 & 8400 & 8500 \\
\hline $\begin{array}{l}\text { Berat Abu Batu (W3 = W2- } \\
\text { W1) }\end{array}$ & $\mathrm{gr}$ & 4300 & 4600 & 4700 \\
\hline Volume Moulding (V) & $\mathrm{cm} 3$ & 3000 & 3000 & 3000 \\
\hline $\begin{array}{l}\text { Berat Volume = (W2- } \\
\text { W1)/V }\end{array}$ & $\mathrm{gr} / \mathrm{cm} 3$ & 1,43 & 1,53 & 1,57 \\
\hline Berat Volume Rata-Rata & $\mathrm{gr} / \mathrm{cm} 3$ & \multicolumn{3}{|c|}{$\mathbf{1 , 5 1}$} \\
\hline
\end{tabular}

Table 3

Sieve Analysis (ASTM C 136-95 A)

\begin{tabular}{|c|c|c|c|c|}
\hline Sieve & Weight & \multicolumn{2}{|c|}{ Retain } & Passing \\
\cline { 1 - 5 } Size & Retain & \multicolumn{2}{|c|}{} & Cumulative \\
\hline (mm) & (gr) & \multicolumn{2}{|c|}{$(\%)$} & $(\%)$ \\
\hline 37,50 & 0 & 0 & 0 & 100 \\
\hline 25,40 & 0 & 0 & 0 & 100 \\
\hline 19,50 & 0 & 0 & 0 & 100 \\
\hline 12,50 & 0 & 0 & 0 & 100 \\
\hline
\end{tabular}




\begin{tabular}{|c|c|c|c|c|}
\hline 9,50 & 0 & 0 & 0 & 100 \\
\hline 4,75 & 0 & 0 & 0 & 100 \\
\hline 2,38 & 406 & 40,60 & 40,60 & 59,40 \\
\hline 1,19 & 374 & 37,40 & 78,00 & 22,00 \\
\hline 0,60 & 163 & 16,30 & 94,30 & 5,70 \\
\hline 0,30 & 42 & 4,20 & 98,50 & 1,50 \\
\hline 0,15 & 1 & 0,10 & 98,60 & 1,40 \\
\hline 0,08 & 10 & 1,00 & 99,60 & 0,40 \\
\hline Pan & 4 & 0,40 & 100 & 0 \\
\hline Total & $1.000,0$ & & & \\
\hline & $\mathrm{Fm}=$ & 4,10 & & \\
\hline
\end{tabular}

Table 4

Specific Gravity Analysis (ASTM C 128-93)

\begin{tabular}{|l|c|c|c|c|}
\hline \multicolumn{1}{|c|}{ PERCOBAAN } & SAT & 1 & 2 & Rata-rata \\
\hline $\begin{array}{l}\text { Berat Abu Batu kering permukaan } \\
\text { jenuh (SSD) }\end{array}$ & $\mathrm{gr}$ & 500 & 500 & 500 \\
\hline Berat Labu ukur + Air (B) & $\mathrm{gr}$ & 1029 & 1029 & 1029 \\
\hline $\begin{array}{l}\text { Berat Labu ukur + Abu Batu (SSD) + } \\
\text { Air (Bt) }\end{array}$ & $\mathrm{gr}$ & 1333 & 1334 & 1333,5 \\
\hline Berat Jenis Kering Permukaan Jenuh $=$ & $\mathrm{gr} / \mathrm{cm} 3$ & 2,551 & 2,564 & $\mathbf{2 , 5 5 8}$ \\
\hline 500/(B+500-Bt) & &
\end{tabular}

Table 5

Infiltration Rate Analysis (ASTM C 128-93)

\begin{tabular}{|l|c|c|}
\hline \multicolumn{1}{|c|}{ PERCOBAAN } & SATUAN & HASIL \\
\hline $\begin{array}{l}\text { Berat Awal Abu Batu kering permukaan } \\
\text { jenuh }(\mathrm{A})\end{array}$ & $\mathrm{gr}$ & 500 \\
\hline Berat kering oven $(\mathrm{B})$ & $\mathrm{gr}$ & 475 \\
\hline Resapan $=(\mathrm{A}-\mathrm{B}) / \mathrm{B}^{*} 100$ & $\%$ & $\mathbf{5 , 2 6}$ \\
\hline
\end{tabular}

Table 6

Ash Stone Cleanliness Analysis of Mud (ASTM C 117)

\begin{tabular}{|l|c|c|}
\hline \multicolumn{1}{|c|}{ PERCOBAAN A (TANPA DICUCI) } & SATUAN & HASIL \\
\hline Berat Abu Batu kering (W1) & gr & 1000 \\
\hline Berat Abu Batu bersih kering (W2) & gr & 863 \\
\hline Kadar Lumpur = (W1-W2)/W1 ${ }^{*} 100$ & $\%$ & $\mathbf{1 3 , 7 0}$ \\
\hline $\begin{array}{c}\text { PERCOBAAN B ( DICUCI 4 - 5 KALI } \\
\text { DALAM MIXER MOLEN ) }\end{array}$ & SATUAN & HASIL \\
\hline Berat Abu Batu kering (W1) & gr & 1000 \\
\hline Berat Abu Batu bersih kering (W2) & gr & 988 \\
\hline Kadar Lumpur = (W1-W2)/W1*100 & $\%$ & $\mathbf{1 , 2 0}$ \\
\hline
\end{tabular}


Compressive Strength Testing

Table 7

Concrete Compressive Strength Test Results Normal

\begin{tabular}{|c|c|c|c|c|c|c|c|c|c|}
\hline \multirow{2}{*}{$\begin{array}{c}\text { Nama } \\
\text { Trial }\end{array}$} & \multicolumn{3}{|c|}{7 Hari } & \multicolumn{3}{|c|}{14 Hari } & \multicolumn{3}{|c|}{28 Hari } \\
\hline & $\mathrm{KN}$ & $\mathrm{Kg} / \mathrm{cm} 2$ & Мра & KN & $\mathrm{Kg} / \mathrm{cm} 2$ & Мра & $\mathrm{KN}$ & $\mathrm{Kg} / \mathrm{cm} 2$ & Мpa \\
\hline \multirow{3}{*}{$\begin{array}{c}\text { BETON } \\
\text { NORMAL } \\
\text { K-300 }\end{array}$} & 332 & \multirow{3}{*}{221,010} & \multirow{3}{*}{18,34} & 398 & \multirow{3}{*}{262,942} & \multirow{3}{*}{21,82} & 440 & \multirow{3}{*}{305,80} & \multirow{3}{*}{25,38} \\
\hline & 316 & & & 367 & & & 445 & & \\
\hline & 306 & & & 370 & & & 435 & & \\
\hline
\end{tabular}

Table 8

Lightweight Concrete Compression Strength Test Results (1)

\begin{tabular}{|c|c|c|c|c|c|c|c|c|c|c|c|}
\hline \multirow{2}{*}{ FOAM } & \multirow{2}{*}{ No } & \multirow{2}{*}{$\begin{array}{l}\text { Nama } \\
\text { Trial }\end{array}$} & \multicolumn{3}{|c|}{7 Hari } & \multicolumn{3}{|c|}{14 Hari } & \multicolumn{3}{|c|}{28 Hari } \\
\hline & & & KN & $\mathrm{Kg} / \mathrm{cm} 2$ & Mpa & KN & $\mathrm{Kg} / \mathrm{cm} 2$ & Mpa & $\mathrm{KN}$ & $\mathrm{Kg} / \mathrm{cm} 2$ & Mpa \\
\hline \multirow{12}{*}{$\begin{array}{c}1: 20 \\
\text { (1 Ilter } \\
\text { FOAM } \\
+20 \\
\text { liter } \\
\text { AIR) }\end{array}$} & \multirow{3}{*}{1} & BRMT 1 & 33 & \multirow{3}{*}{33,128} & \multirow{3}{*}{2,75} & 86 & \multirow{3}{*}{76,450} & \multirow{3}{*}{6,35} & 136 & \multirow{3}{*}{92,203} & \multirow{3}{*}{7,65} \\
\hline & & (BO & 34 & & & 111 & & & 105 & & \\
\hline & & $\begin{array}{l}0,5 \% \\
\text { SF } 5 \%)\end{array}$ & 76 & & & 133 & & & 157 & & \\
\hline & \multirow{3}{*}{2} & BRMT 2 & $\frac{111}{71}$ & \multirow{3}{*}{80,157} & \multirow{3}{*}{6,65} & 174 & \multirow{3}{*}{114,212} & \multirow{3}{*}{9,48} & $\frac{131}{176}$ & \multirow{3}{*}{126,722} & \multirow{3}{*}{10,52} \\
\hline & & $\begin{array}{r}\text { (BO } \\
05 \%\end{array}$ & 74 & & & 98 & & & 176 & & \\
\hline & & $\begin{array}{c}\text { SF } \\
10 \%) \\
\end{array}$ & 161 & & & 221 & & & 240 & & \\
\hline & \multirow{3}{*}{3} & BRMT 3 & 138 & \multirow{3}{*}{82,473} & \multirow{3}{*}{6,85} & 186 & \multirow{3}{*}{102,860} & \multirow{3}{*}{8,54} & 199 & \multirow{3}{*}{121,857} & \multirow{3}{*}{10,11} \\
\hline & & (BO $1 \%$, & 115 & & & 118 & & & 175 & & \\
\hline & & SF 5\%) & 103 & & & 140 & & & 152 & & \\
\hline & \multirow{3}{*}{4} & BRMT 4 & 103 & \multirow{3}{*}{67,647} & \multirow{3}{*}{5,61} & 86 & \multirow{3}{*}{68,573} & \multirow{3}{*}{5,69} & 134 & \multirow{3}{*}{89,192} & \multirow{3}{*}{7,40} \\
\hline & & (B0 1\%, & 91 & & & 111 & & & 113 & & \\
\hline & & SF & 98 & & & 99 & & & 138 & & \\
\hline
\end{tabular}

Table 9

Lightweight Concrete Compression Strength Test Results (2)

\begin{tabular}{|c|c|c|c|c|c|c|c|c|c|c|c|}
\hline \multirow{2}{*}{ FOAM } & \multirow{2}{*}{ No } & \multirow{2}{*}{$\begin{array}{c}\text { Nama } \\
\text { Trial }\end{array}$} & \multicolumn{3}{|c|}{7 Hari } & \multicolumn{3}{|c|}{14 Hari } & \multicolumn{3}{|c|}{28 Hari } \\
\hline & & & KN & $\mathrm{Kg} / \mathrm{cm} 2$ & Mpa & KN & $\mathrm{Kg} / \mathrm{cm} 2$ & Mpa & KN & $\mathrm{Kg} / \mathrm{cm} 2$ & Mpa \\
\hline \multirow{9}{*}{$\begin{array}{l}1: 40 \\
\text { (1 Ilter } \\
\text { FOAM } \\
+40 \\
\text { liter } \\
\text { AIR) }\end{array}$} & \multirow{3}{*}{1} & \multirow{3}{*}{$\begin{array}{c}\text { ULANG } \\
\text { BRMT } \\
1 \text { (BO } \\
0,5 \% \text {, } \\
\text { SF 5\%) }\end{array}$} & 126 & \multirow{3}{*}{95,910} & \multirow{3}{*}{7,96} & 160 & \multirow{3}{*}{116,992} & \multirow{3}{*}{9,71} & 182 & \multirow{3}{*}{132,945} & \multirow{3}{*}{11,03} \\
\hline & & & 148 & & & 193 & & & 219 & & \\
\hline & & & 140 & & & 152 & & & 173 & & \\
\hline & \multirow[b]{3}{*}{2} & \multirow{3}{*}{$\begin{array}{c}\text { ULANG } \\
\text { BRMT } \\
2 \text { (BO } \\
0,5 \%, \\
\text { SF } \\
10 \%) \\
\end{array}$} & 199 & \multirow[b]{3}{*}{136,915} & \multirow[b]{3}{*}{11,36} & 244 & \multirow[b]{3}{*}{167,727} & \multirow[b]{3}{*}{13,92} & 277 & \multirow[b]{3}{*}{190,598} & \multirow[b]{3}{*}{15,82} \\
\hline & & & 203 & & & 241 & & & 274 & & \\
\hline & & & 189 & & & 239 & & & 272 & & \\
\hline & \multirow{3}{*}{3} & \multirow{3}{*}{$\begin{array}{c}\text { ULANG } \\
\text { BRMT } \\
3 \text { (BO } \\
1 \%, \text { SF } \\
5 \%)\end{array}$} & 176 & \multirow{3}{*}{120,235} & \multirow{3}{*}{9,98} & 188 & \multirow{3}{*}{127,648} & \multirow{3}{*}{10,59} & 214 & \multirow{3}{*}{145,055} & \multirow{3}{*}{12,04} \\
\hline & & & 169 & & & 161 & & & 183 & & \\
\hline & & & 174 & & & 202 & & & 230 & & \\
\hline
\end{tabular}




\begin{tabular}{|c|c|c|c|c|c|c|c|c|c|c|}
\hline \multirow{3}{*}{\multicolumn{2}{|c|}{$\begin{array}{c}\text { ULANG } \\
\text { BRMT } \\
4 \text { (B0 } \\
1 \%, \text { SF } \\
10 \%)\end{array}$}} & 168 & \multirow{3}{*}{111,200} & \multirow{3}{*}{9,23} & 200 & \multirow{3}{*}{131,355} & \multirow{3}{*}{10,90} & 227 & \multirow{3}{*}{149,267} & \multirow{3}{*}{12,39} \\
\hline & & 160 & & & 184 & & & 209 & & \\
\hline & & 152 & & & 183 & & & 208 & & \\
\hline
\end{tabular}

Based on the table above, the highest quality is found in 1:40 ratio variation (foam: water) using Silica Fume $10 \%$ and $0.5 \%$ Bonding which achieves quality $\mathrm{K} 191 \mathrm{~kg} / \mathrm{cm} 2$ or FC $10.82 \mathrm{MPa}$.

Table 10

Normal Concrete Specific Gravity

\begin{tabular}{|c|c|c|c|c|c|c|c|}
\hline \multirow{2}{*}{$\begin{array}{c}\text { Nama } \\
\text { Trial }\end{array}$} & \multicolumn{2}{|c|}{7 Hari } & \multicolumn{2}{|c|}{14 Hari } & \multicolumn{2}{|c|}{28 Hari } & \multirow{2}{*}{$\begin{array}{c}\text { Rata-Rata } \\
\mathrm{Kg} / \mathrm{m} 3\end{array}$} \\
\hline & Berat & $\begin{array}{l}\text { Density } \\
\text { Aktual } \\
\mathrm{Kg} / \mathrm{m} 3 \\
\end{array}$ & Berat & $\begin{array}{l}\text { Density } \\
\text { Aktual } \\
\mathrm{Kg} / \mathrm{m} 3 \\
\end{array}$ & Berat & $\begin{array}{l}\text { Density } \\
\text { Aktual } \\
\mathrm{Kg} / \mathrm{m} 3 \\
\end{array}$ & \\
\hline \multirow{3}{*}{$\begin{array}{c}\text { BETON } \\
\text { NORMAL } \\
\text { K-300 }\end{array}$} & 12,7 & \multirow{3}{*}{2403} & 12,6 & \multirow{3}{*}{2390} & 12,7 & \multirow{3}{*}{2409} & \multirow{3}{*}{2400,419} \\
\hline & 12,7 & & 12,7 & & 12,8 & & \\
\hline & 12,8 & & 12,7 & & 12,8 & & \\
\hline
\end{tabular}

Table 11

Lightweight Average Concrete Species

\begin{tabular}{|c|c|c|c|c|c|c|c|c|}
\hline \multirow[b]{2}{*}{ No } & \multirow[b]{2}{*}{ Nama Trial } & \multicolumn{2}{|c|}{7 Hari } & \multicolumn{2}{|c|}{14 Hari } & \multicolumn{3}{|c|}{28 Hari } \\
\hline & & $\begin{array}{c}\text { Berat } \\
\text { Kg }\end{array}$ & $\begin{array}{l}\text { Density } \\
\text { Aktual } \\
\text { Kg/m3 }\end{array}$ & $\begin{array}{c}\text { Berat } \\
\text { kg }\end{array}$ & $\begin{array}{l}\text { Density } \\
\text { Aktual } \\
\text { Kg/m3 }\end{array}$ & $\begin{array}{c}\text { Berat } \\
\text { Kg }\end{array}$ & $\begin{array}{c}\text { Density } \\
\text { Aktual } \\
\mathrm{Kg} / \mathrm{m} 3\end{array}$ & $\begin{array}{c}\text { Density } \\
\text { Rata-Rata } \\
\mathrm{Kg} / \mathrm{m} 3\end{array}$ \\
\hline \multirow{3}{*}{1} & \multirow{3}{*}{$\begin{array}{l}\text { BRMT } 1 \text { (BO } \\
0,5 \%, \text { SF } 5 \%)\end{array}$} & 8,2 & \multirow{3}{*}{1629} & 8 & \multirow{3}{*}{1692} & 9,2 & \multirow{3}{*}{1673} & \\
\hline & & 8,7 & & 9,5 & & 8,4 & & \\
\hline & & 9 & & 9,4 & & 9 & & \\
\hline \multirow{3}{*}{2} & \multirow{3}{*}{$\begin{array}{c}\text { BRMT } 2 \text { (BO } \\
0,5 \%, \text { SF } 10 \%)\end{array}$} & 9,8 & \multirow{3}{*}{1811} & 10,5 & \multirow{3}{*}{1805} & 9,6 & \multirow{3}{*}{1811} & \\
\hline & & 8,6 & & 9 & & 8,9 & & \multirow{8}{*}{1722} \\
\hline & & 10,4 & & 9,2 & & 10,3 & & \\
\hline \multirow{3}{*}{3} & \multirow{3}{*}{$\begin{array}{l}\text { BRMT } 3(\mathrm{BO} \\
1 \%, \text { SF } 5 \%)\end{array}$} & 9 & \multirow{3}{*}{1742} & 9,5 & \multirow{3}{*}{1792} & 9 & \multirow{3}{*}{1717} & \\
\hline & & 9,4 & & 9,4 & & 9,3 & & \\
\hline & & 9,3 & & 9,6 & & 9 & & \\
\hline \multirow{3}{*}{4} & \multirow{3}{*}{$\begin{array}{l}\text { BRMT } 4(\text { B0 1\%, } \\
\text { SF 10\%) }\end{array}$} & 8,4 & \multirow{3}{*}{1623} & 8,6 & \multirow{3}{*}{1686} & 8,8 & \multirow{3}{*}{1679} & \\
\hline & & 8,3 & & 9,2 & & 9,1 & & \\
\hline & & 9,1 & & 9 & & 8,8 & & \\
\hline \multirow{3}{*}{5} & \multirow{3}{*}{$\begin{array}{c}\text { ULANG BRMT } 1 \\
\text { (BO 0,5\%, SF } \\
5 \%)\end{array}$} & 9,8 & \multirow{3}{*}{1862} & 10 & \multirow{3}{*}{1893} & 10 & \multirow{3}{*}{1893} & \multirow{6}{*}{1884} \\
\hline & & 9,9 & & 10 & & 10,1 & & \\
\hline & & 9,9 & & 10,1 & & 10 & & \\
\hline \multirow{3}{*}{6} & \multirow{3}{*}{$\begin{array}{c}\text { ULANG BRMT } 2 \\
\text { (BO 0,5\%, SF } \\
10 \%)\end{array}$} & 10 & \multirow{3}{*}{1893} & 10 & & 10 & & \\
\hline & & 10,1 & & 10,1 & 1899 & 10,1 & 1899 & \\
\hline & & 10 & & 10,1 & & 10,1 & & \\
\hline
\end{tabular}




\begin{tabular}{|c|c|c|c|c|c|c|c|}
\hline \multirow{3}{*}{7} & \multirow{3}{*}{$\begin{array}{l}\text { ULANG BRMT } 3 \\
\text { (BO } 1 \%, \text { SF } 5 \%)\end{array}$} & 9,9 & \multirow{3}{*}{1881} & 10 & \multirow{3}{*}{1893} & 10,1 & \multirow{3}{*}{1893} \\
\hline & & 10 & & 10,1 & & 10 & \\
\hline & & 10 & & 10 & & 10 & \\
\hline \multirow{3}{*}{8} & \multirow{3}{*}{$\begin{array}{c}\text { ULANG BRMT } 4 \\
\text { (B0 1\%, SF } \\
10 \%)\end{array}$} & 9,7 & \multirow{3}{*}{1836} & 9,9 & \multirow{3}{*}{1881} & 9,9 & \multirow{3}{*}{1881} \\
\hline & & 9,7 & & 10 & & 10 & \\
\hline & & 9,8 & & 10 & & 10 & \\
\hline
\end{tabular}

Based on the results of the trial obtained an average density or specific gravity above in accordance with the requirements of $<1900 \mathrm{~kg} / \mathrm{m}^{3}$, in the first trial with a foam ratio of 1:20 obtained an average of $1722 \mathrm{~kg} / \mathrm{m}^{3}$ and a second trial of foam ratio of 1:40 obtained an average of an average of $1884 \mathrm{~kg} / \mathrm{m}^{3}$.

\section{CONCLUSION}

On the results of research and discussion that has been carried out several conclusions can be drawn as follows:

1. The use of ash stone material and foam agent (foam) in this study was able to produce concrete weighing $<1900 \mathrm{~kg} / \mathrm{m}^{3}$, as evidenced in the average results in the first trial mix with the ratio of foam: water $(1: 20)$ to get the result of specific gravity $1722 \mathrm{~kg} / \mathrm{m}^{3}$ and the second trial mix with a ratio of foam: water (1:40) yielding a specific gravity of $1884 \mathrm{~kg} / \mathrm{m}^{3}$.

2. The addition of silica fume, superplasticizer (type f), and bonding can affect quality but do not reach the K-300 quality target. In this study the addition of optimum silica fume at a proportion of $10 \%$, the more additions it will add to the strength of concrete. The addition of type $f$ in this study is optimum in the proportion of $0.6 \%$ because it can reduce water use by $12 \%$. Whereas the addition of optimum bonding is in the proportion of $0.5 \%$ because if the bonding is mixed with foam / foam even though the proportion added does not really affect the strength of the concrete.

3. In this research, normal concrete with K-300 quality compared to lightweight concrete whose composition is cement, stone ash, silica fume, type $f$, and bonding. The results of normal concrete compressive strength obtained quality $\mathrm{K} 305.80 \mathrm{~kg} / \mathrm{cm}^{2}$ or FC $25.38 \mathrm{MPa}$, whereas for lightweight concrete obtained with a foam: water composition ratio (1:40), the proportion of silica fume use $10 \%, 0.5 \%$ bonding with compressive strength of $\mathrm{K} 190,6 \mathrm{~kg} / \mathrm{cm}^{2}$ or FC $15,82 \mathrm{Mpa}$. In each specimen the value of quality efficiency with the most optimum weight variation is SF $10 \%$; BO $0.5 \%$ with a coefficient value of $10.03 \%$.

\section{SUGGESTION}

In order to produce research that will later become better in further research the authors suggest paying attention to the following matters:

1. There needs to be another trial and error in order to get the right lightweight concrete mixing and the quality to reach the desired target;

2. Use stone ash which has a uniform grain size and has a standard mud content by washing it first;

3. Add additives and admixture that can reduce more water so that the quality of concrete is in accordance with the target;

4. Pay attention to the moisture content test for the material because it affects the use of the amount of water in the concrete mixture;

5. Pay attention to the density foam to fit the requirements of $0.055 \mathrm{t} / \mathrm{m}^{3}-0.085 \mathrm{t} / \mathrm{m}^{3}$. 


\section{REFERENCES}

[1]. Jitchaiyaphum, K. (2011), Cellular Lightweight Concrete Containing Pozzolan Materials, Science Direct, Elsevier Ltd.

[2]. Mulyono, T. (2004). Teknologi Beton, Yogyakarta: Andi

[3]. ASTM Standart C 109. (2002). Standard Test Method for Compressive Strength of Hydraulic Cement Mortars. USA: ASTM International.

[4]. ASTM Standart C 117 (2003). Test Method for Materials Finer than 75-m (No. 200) Sieve in Mineral Aggregates by Washing (ASTM C 117-95). USA: ASTM International.

[5]. $\quad$ ASTM Standart C 29. (2002). Test Method for Bulk Density (Unit Weight) and Voids in Aggregate (ASTM C 29/C 29 M-91a). USA: ASTM International.

[6]. ASTM Standart C 31. (2002). Standart Practice Making and Curing Concrete test specimens in field (ASTM C 31-09). USA: ASTM International.

[7]. ASTM Standart C 33. (1992). Standard Specification for Concrete Aggregate (ASTM C33-92). West Conshohocken, PA, USA: ASTM International.

[8]. ASTM Standart C 348. (2005). Standar Tests Method for Flexural Strength of Hydraulic Cement Mortar (ASTM C 348-97 S). West Conshohocken, PA, USA: ASTM International.

[9]. ASTM Standart C 40. Standard Test Method for Organic Impurities in Fine Aggregates for Concrete (ASTM C 40-92). United States.

[10]. ASTM Standart C 494. (2002). Standart Specifications For Chemical Admixtures For Concrete (ASTM C 494-86). USA: ASTM International

[11]. ASTM.C.1240, 637-642. (1995). Spesification for Silica Fume faor Use in Hydraulic Cement Concrete and Mortal. USA: ASTM International

[12]. PBI (1971), Peraturan Beton Bertulang Indonesia (PBI-1971), Bandung: Departemen Pekerjaan Umum

[13]. SII 0013. (1981). Mutu dan Cara Uji Semen Portland (SII 0013-81). Jakarta: Departemen Perindustrian Republik Indonesia 